\title{
Spectrum of the SU(3) Dirac operator on the lattice: Transition from random matrix theory to chiral perturbation theory
}

\author{
M. Göckeler, H. Hehl, P. E. L. Rakow, A. Schäfer \\ Institut für Theoretische Physik, Universität Regensburg, D-93040 Regensburg, Germany \\ T. Wettig \\ Department of Physics, Yale University, New Haven, CT 06520-8120, USA \\ RIKEN-BNL Research Center, Brookhaven National Laboratory, Upton, NY 11973-5000, USA
}

(May 14, 2000)

\begin{abstract}
We calculate complete spectra of the Kogut-Susskind Dirac operator on the lattice in quenched $\mathrm{SU}(3)$ gauge theory for various values of coupling constant and lattice size. From these spectra we compute the connected and disconnected scalar susceptibilities and find agreement with chiral random matrix theory up to a certain energy scale, the Thouless energy. The dependence of this scale on the lattice volume is analyzed. In the case of the connected susceptibility this dependence is anomalous, and we explain the reason for this. We present a model of chiral perturbation theory that is capable of describing the data beyond the Thouless energy and that has a common range of applicability with chiral random matrix theory.
\end{abstract}

PACS numbers: 11.15.Ha, 05.40.-a, 11.30.Rd, 12.38.Gc

\section{INTRODUCTION}

It has by now been well established that the low-lying eigenvalues of the lattice QCD Dirac operator can be described by chiral random matrix theory (chRMT) [1] if the linear size $L$ of a lattice with Euclidean 4 -volume $V=L^{4}$ fulfills the constraints

$$
1 / \Lambda \ll L \ll 1 / m_{\pi},
$$

where $\Lambda$ is a typical hadronic scale such as the rho mass, and $m_{\pi}$ is the pion mass [2, 3]. The first inequality means that the low-energy features of the theory can be described in terms of Goldstone modes governed by an effective chiral Lagrangian, whereas the second inequality tells us that the zero-momentum modes make the dominant contribution to the partition function so that the kinetic terms in the chiral Lagrangian can be neglected. This implies that in the phase where chiral symmetry is spontaneously broken the low-lying eigenvalues of the Dirac operator are only sensitive to symmetry properties of the underlying theory. For staggered fermions one finds that the chiral Gaussian symplectic ensemble (chGSE) of chRMT corresponds to SU(2) gauge theory with fermions in the fundamental representation, the chiral Gaussian orthogonal ensemble (chGOE) is relevant for $\mathrm{SU}\left(N_{c}\right), N_{c} \geq 2$, gauge theory with adjoint fermions, and the chiral Gaussian unitary ensemble (chGUE) describes $\operatorname{SU}\left(N_{c}\right), N_{c} \geq 3$, gauge theory with fundamental fermions [4,5]. Agreement with chRMT has been confirmed for all three ensembles, primarily for the microscopic spectral density of the Dirac operator [5 10].

In earlier papers 11 14 the energy scale up to which chRMT applies, the so-called Thouless energy, has been predicted theoretically and verified in $\mathrm{SU}(2)$ lattice simulations. In these investigations it has been found that this upper energy scale $\lambda_{\mathrm{RMT}}$ is given by

$$
\lambda_{\mathrm{RMT}} / \Delta \sim f_{\pi}^{2} L^{2},
$$

where $\Delta=1 / \rho(0)=\pi /(V \Sigma)$ is the mean level spacing at virtuality zero as given by the Banks-Casher formula. Here, $f_{\pi}$ is the pion decay constant (normalized such that $f_{\pi}=93 \mathrm{MeV}$ in the real world), $\Sigma$ is the absolute value of the 4-flavor chiral condensate for infinite volume and vanishing mass, and $\rho(\lambda)$ is the spectral density of the Dirac operator averaged over gauge field configurations.

In this paper we extend our analysis of the Thouless energy to the physically more interesting case of SU(3). We concentrate on the chiral susceptibilities $\chi^{\text {disc }}$ and $\chi^{\text {conn }}$ which in the $\mathrm{SU}(2)$ case were shown to be especially well suited for such an analysis [14, 15]. For an earlier analysis of $\chi^{\text {disc }}$ in the $\mathrm{SU}(3)$ case and a general comparison of chRMT to our data we refer to Ref. 10. In order to describe the Dirac spectrum beyond the Thouless energy, we then construct a model of chiral perturbation theory that takes into account the differences between KogutSusskind fermions at finite lattice spacing and continuum fermions. A similar analysis using continuum chiral perturbation theory was performed in the framework of an instanton liquid model in Ref. [12].

\section{SIMULATIONS}

The Euclidean Kogut-Susskind Dirac operator on the lattice reads

$$
\not D_{x, y}=\frac{1}{2} \sum_{\mu}\left[\eta_{\mu}(x) U_{\mu}(x) \delta_{x+\hat{\mu}, y}-\eta_{\mu}(y) U_{\mu}^{\dagger}(y) \delta_{x-\hat{\mu}, y}\right]
$$

where $U$ and $\eta$ denote the link variables and the staggered phases, respectively, and the lattice spacing has been set 


\begin{tabular}{ccccc}
\hline \hline$\beta \backslash V$ & $4^{4}$ & $6^{4}$ & $8^{4}$ & $10^{4}$ \\
\hline 5.2 & 40830 & 24708 & 7665 & 5806 \\
5.4 & 35337 & 24210 & 6000 & 6300 \\
5.6 & 36158 & 21000 & 8000 & 7700 \\
5.7 & & 19000 & 6000 & 6198 \\
\hline \hline
\end{tabular}

TABLE I. Number of generated configurations.

\begin{tabular}{cllll}
\hline \hline$\beta \backslash V$ & \multicolumn{1}{c}{$4^{4}$} & \multicolumn{1}{c}{$6^{4}$} & \multicolumn{1}{c}{$8^{4}$} & \multicolumn{1}{c}{$10^{4}$} \\
\hline 5.2 & $1.08(4)$ & $1.06(3)$ & $1.06(4)$ & $1.07(4)$ \\
5.4 & $0.849(30)$ & $0.875(20)$ & $0.86(2)$ & $0.87(2)$ \\
5.6 & $0.47(10)$ & $0.47(2)$ & $0.47(2)$ & $0.47(3)$ \\
5.7 & & $0.255(80)$ & $0.242(40)$ & $0.255(50)$ \\
\hline \hline
\end{tabular}

TABLE II. Absolute values of the chiral condensate $\Sigma$.

equal to one, i.e., we use lattice units. The Dirac operator in Eq. (3) is anti-hermitian. Hence its eigenvalues $i \lambda_{k}$ are purely imaginary $\left(\lambda_{k} \in \mathbb{R}\right)$. Furthermore, the spectrum is symmetric about zero, i.e., for each eigenvalue $\lambda_{k} \neq 0$ there is another eigenvalue $-\lambda_{k}$. Note that for $N_{c} \geq 3$ the eigenvalues are generically non-degenerate, other than in $\mathrm{SU}(2)$ where each eigenvalue is doubly degenerate. We compute spectra of $\not D$ using the same methods as in Ref. [10].

Table 1 gives the number of configurations for the values of $\beta=6 / g^{2}$ (with $g$ the coupling constant) and the lattice volume $L^{4}$ that we used. The absolute values of the (unrenormalized) chiral condensate $\Sigma$ together with the statistical errors are given in Table III. They were computed from a fit of the distribution of the smallest positive eigenvalue $\lambda_{\min }$ to the chRMT formula 16] $P\left(\lambda_{\min }\right)=\frac{1}{2}(V \Sigma)^{2} \lambda_{\min } \mathrm{e}^{-\left(V \Sigma \lambda_{\min }\right)^{2} / 4}$.

\section{SCALAR SUSCEPTIBILITIES}

Consider a lattice theory with two kinds of quarks, a valence quark with mass $m_{v}$ and a sea quark with mass $m_{s}$. We will take $N_{v}$ generations of valence quarks and $N_{s}$ generations of sea quarks. Each generation corresponds to 4 flavors in the continuum limit. The partition function $Z$ for the theory is given as a sum over gauge field configurations $U$,

$$
Z\left(m_{v}, m_{s}\right)=\sum_{U} \mathrm{e}^{-S_{g}(U)} \operatorname{det}\left(m_{v}+\not D\right)^{N_{v}} \operatorname{det}\left(m_{s}+\not D\right)^{N_{s}},
$$

where $S_{g}(U)$ is the gauge action. The definitions of the chiral condensate $\sigma$ and the two scalar susceptibilities for $N_{c} \geq 3$ are then

$$
\sigma\left(m_{v}, m_{s}\right)=\lim _{N_{v} \rightarrow 0} \frac{1}{V N_{v}} \frac{\partial}{\partial m_{v}} \ln Z\left(m_{v}, m_{s}\right),
$$

$$
\begin{aligned}
\chi^{\operatorname{conn}}\left(m_{v}\right) & =\left.\frac{\partial}{\partial m_{v}} \sigma\left(m_{v}, m_{s}\right)\right|_{m_{s}=m_{v}}, \\
\chi^{\operatorname{disc}}\left(m_{v}\right) & =\left.\frac{1}{N_{s}} \frac{\partial}{\partial m_{s}} \sigma\left(m_{v}, m_{s}\right)\right|_{m_{s}=m_{v}} .
\end{aligned}
$$

For quenched $\left(N_{s}=0\right)$ Kogut-Susskind fermions $\sigma$ depends only on the valence quark mass $m_{v}=m$. From the complete spectra of $\not D$ we compute $\sigma$ for arbitrary values of $m$ according to

$$
\sigma_{\text {lattice }}(m)=\frac{1}{V}\left\langle\sum_{k=1}^{N} \frac{1}{\mathrm{i} \lambda_{k}+m}\right\rangle,
$$

where $N=N_{c} V$ is the number of the eigenvalues $\mathrm{i} \lambda_{k}$ of $\not D$ and the average is over gauge field configurations. The susceptibilities are given by

$$
\chi_{\text {lattice }}^{\text {conn }}(m)=\frac{\partial}{\partial m} \sigma(m)=-\frac{1}{V}\left\langle\sum_{k=1}^{N} \frac{1}{\left(\mathrm{i} \lambda_{k}+m\right)^{2}}\right\rangle
$$

and

$$
\begin{aligned}
\chi_{\text {lattice }}^{\text {disc }}(m)= & \frac{1}{V}\left\langle\sum_{k, \ell=1}^{N} \frac{1}{\left(\mathrm{i} \lambda_{k}+m\right)\left(\mathrm{i} \lambda_{\ell}+m\right)}\right\rangle \\
& -\frac{1}{V}\left\langle\sum_{k=1}^{N} \frac{1}{\mathrm{i} \lambda_{k}+m}\right\rangle^{2} .
\end{aligned}
$$

In the last equation the limit $m_{s}=m_{v}=m$ has been taken after evaluating the derivative in Eq. (7). Note that slightly different definitions are used for gauge group $\mathrm{SU}(2)$ because of the degeneracy of the eigenvalues, see Ref. 115.

From chRMT one obtains expressions for the chiral condensate and the susceptibilities which depend on $N_{s}$ and on the topological charge $\nu$. In order to compare with our numerical data we have to set $N_{s}=0$ because we work in the quenched approximation. We shall also set $\nu=0$, since the staggered Dirac operator has no exact zero modes (not even approximate ones because of the relatively strong couplings we use) so that we are effectively in the sector of vanishing topological charge. Thus we get from the chGUE, which is the appropriate ensemble for the gauge group $\mathrm{SU}(3)$, the following result for the chiral condensate,

$$
\frac{\sigma_{\mathrm{RMT}}}{\Sigma}=u\left[I_{0}(u) K_{0}(u)+I_{1}(u) K_{1}(u)\right],
$$

where the rescaled mass parameter $u$ is given by

$$
u=m \Sigma L^{4} \text {. }
$$

The functions $I_{n}, K_{n}$ are modified Bessel functions. For the connected susceptibility one has

$$
\frac{\chi_{\mathrm{RMT}}^{\mathrm{conn}}}{V \Sigma^{2}}=I_{0}(u) K_{0}(u)-I_{1}(u) K_{1}(u),
$$


and for the disconnected susceptibility

$$
\frac{\chi_{\mathrm{RMT}}^{\mathrm{disc}}}{V \Sigma^{2}}=u^{2}\left[I_{0}^{2}(u)-I_{1}^{2}(u)\right]\left[K_{1}^{2}(u)-K_{0}^{2}(u)\right] .
$$

Once $\Sigma$ has been fixed, the chRMT predictions do not contain any free parameters.

\section{SCALING OF THE THOULESS ENERGY}

In Refs. 14, 15, 17 it was demonstrated for the gauge group $\mathrm{SU}(2)$ that chRMT describes $\chi^{\text {conn }}$ and $\chi^{\text {disc }}$ perfectly up to a value of $u$ which scales like $L^{2}$, in agreement with Eq. (2). The same scaling was found for $\chi^{\text {disc in the }}$ case of SU(3) [10]. Interestingly, this is not true for $\chi^{\text {conn }}$ in SU(3) 17], where we find instead a scaling with $L^{4 / 3}$, see Fig. 11. This surprising behavior can be traced back to the form of the asymptotic expansion of $\chi_{\mathrm{RMT}}^{\mathrm{conn}}$ as we shall now explain.

Let us assume that the susceptibilities can be described by

$$
\chi \approx \chi_{\mathrm{RMT}}+\text { constant } .
$$

(We will present a more sophisticated model in terms of chiral perturbation theory later in this article.) The constant describes the large-mass limit $(u \rightarrow \infty)$ of $\chi$ that will be of order 1 in lattice units. In this limit finite-size effects are negligible, and the constant is therefore also the thermodynamic limit of $\chi$. It will become important when the mass has become so large that $\chi_{\mathrm{RMT}}$ has dropped to values of order 1 .

Now, the asymptotic behavior $(u \rightarrow \infty)$ of the chRMT predictions of the two susceptibilities depends on the color group and is given in Table III. Note the different powers of $u$ in the denominator of $\chi_{\mathrm{RMT}}^{\mathrm{conn}}$.

\begin{tabular}{lll}
\hline \hline & $\mathrm{SU}(2)-$ chGSE & $\mathrm{SU}(3)-$ chGUE \\
\hline$\chi_{\mathrm{RMT}}^{\text {disc }}$ & $\frac{V \Sigma^{2}}{8 u^{2}}+\mathcal{O}\left(u^{-3}\right)$ & $\frac{V \Sigma^{2}}{4 u^{2}}+\mathcal{O}\left(u^{-4}\right)$ \\
$\chi_{\mathrm{RMT}}^{\text {conn }}$ & $\frac{V \Sigma^{2}}{4 u^{2}}+\mathcal{O}\left(u^{-3}\right)$ & $\frac{V \Sigma^{2}}{4 u^{3}}+\mathcal{O}\left(u^{-4}\right)$ \\
\hline
\end{tabular}

TABLE III. Asymptotic expansions of the two susceptibilities with different gauge groups.

In the standard case, where the expansion starts with $1 / u^{2}, \chi$ gets of order 1 at $u^{2} \sim V \Sigma^{2}$, i.e., $u \sim L^{2}$. This is the case of $\mathrm{SU}(2)$ and has a natural explanation, namely, that the Compton wavelength of the pion becomes comparable with the box size $L$ of the lattice. This follows from the upper bound of (1) and Eq. (2). In the case of $\mathrm{SU}(3) \chi^{\text {conn }} \sim 1$ when $u^{3} \sim V \Sigma^{2}$ and so the value of $u$ where RMT breaks down scales with $L^{4 / 3}$ instead.

Where does the unusual power come from? It is a consequence of the quenched formulation and of the fact
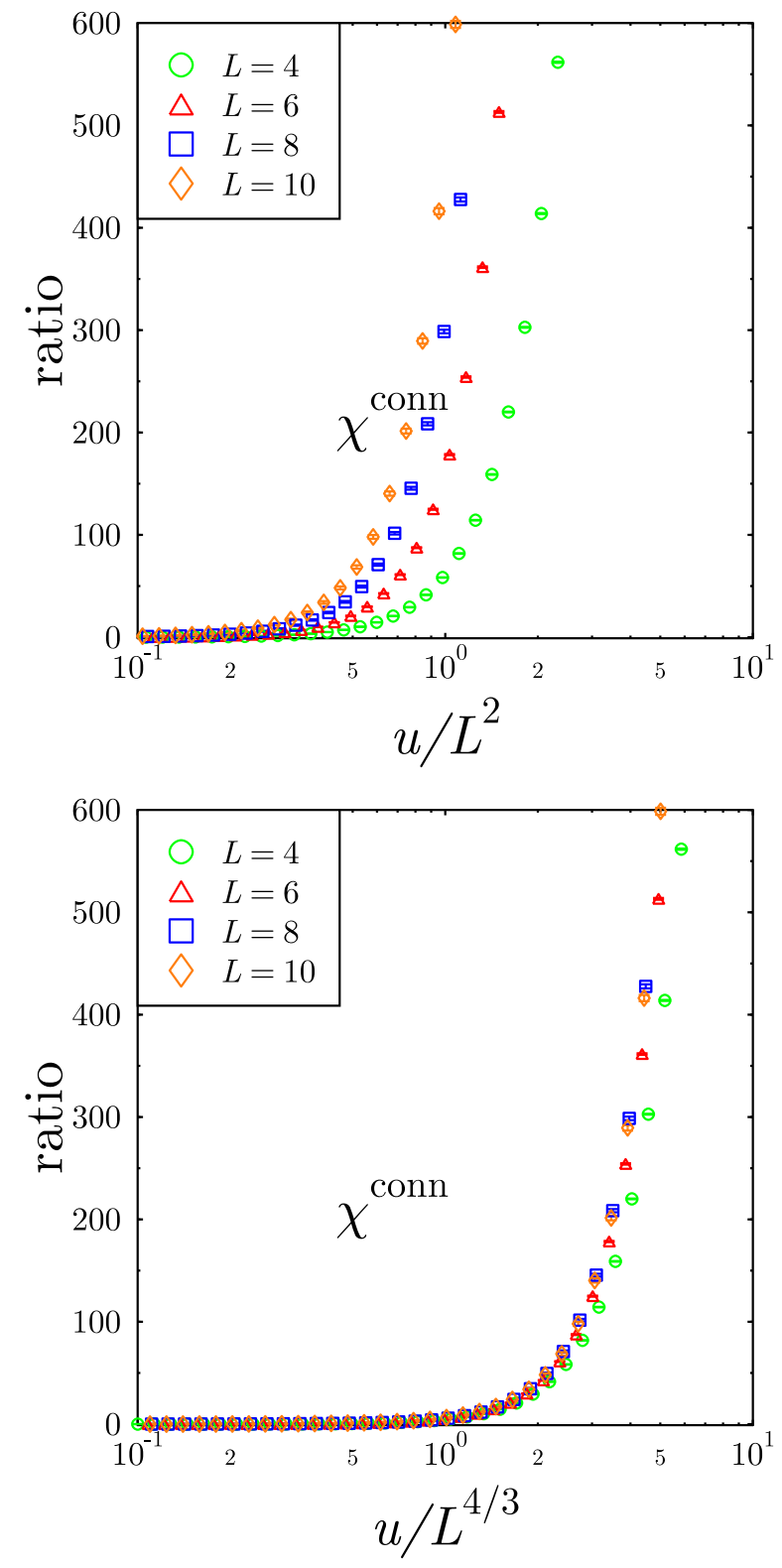

FIG. 1. Ratios $\left(\chi_{\text {lattice }}^{\text {conn }}-\chi_{\mathrm{RMT}}^{\text {conn }}\right) / \chi_{\mathrm{RMT}}^{\text {conn }}$ of the connected scalar susceptibility $\chi^{\text {conn }}$ for $\mathrm{SU}(3)$ staggered fermions at $\beta=5.2$ and various lattice volumes $V=L^{4}$.

that our lattice simulations are effectively in the sector of topological quantum number $\nu=0$. The general chRMT prediction for arbitrary topological quantum number $\nu$ and number of flavors $N_{s}$ is given by

$$
\frac{\chi_{\mathrm{RMT}}^{\mathrm{conn}}}{V \Sigma^{2}}=I_{n}(u) K_{n}(u)-I_{n+1}(u) K_{n-1}(u)
$$

with $n=N_{s}+|\nu|$. This leads to an asymptotic expansion

$$
\frac{\chi_{\mathrm{RMT}}^{\mathrm{conn}}}{V \Sigma^{2}} \sim \frac{n}{u^{2}}-\frac{4 n^{2}-1}{4 u^{3}}+\mathcal{O}\left(u^{-4}\right) .
$$


Thus, unless $N_{s}=\nu=0$ (as in our case) one should recover the usual behavior. It would be very desirable to check this prediction in unquenched lattice simulations $\left(N_{s} \neq 0\right)$ or with Ginsparg-Wilson fermions, which can reproduce the $\nu \neq 0$ sectors of QCD [18].

\section{CHIRAL PERTURBATION THEORY}

We want to describe the lattice data also beyond the Thouless energy and therefore have to use a physical model that goes beyond RMT. One description of the low-energy limit of QCD is chiral perturbation theory (chPT). Since all our data are from quenched simulations we actually have to use the quenched version of chPT.

The data we consider are rather far from the continuum limit, so we cannot rely on the symmetry breaking pattern that is seen in continuum QCD but have to use its lattice version instead. 15

Our starting point is a partition function of the form

$$
\begin{aligned}
\ln Z\left(m_{v}, m_{s}\right) \propto & V S\left(m_{v}, m_{s}\right) \\
& -\frac{1}{2} \sum_{Q} K_{Q} \sum_{p} \ln \left[\hat{p}^{2}+m_{Q}^{2}\left(m_{v}, m_{s}\right)\right] .
\end{aligned}
$$

$S\left(m_{v}, m_{s}\right)$ is the saddle-point contribution leading to a smooth background, and the double sum represents the one-loop contribution due to light composite (Goldstone) bosons. The sum runs over the allowed lattice momenta $p_{\mu}\left[p_{\mu}=2 \pi n_{\mu} / L\right.$ with integer $n_{\mu}$ and $\hat{p}^{2} \equiv 2 \sum_{\mu}(1-$ $\left.\cos p_{\mu}\right)$ ] as well as over light particles of type $Q$ with multiplicity $K_{Q}$ and mass $m_{Q}$.

In order to calculate the values for $K_{Q}$ and $m_{Q}$ we need to know the pattern of chiral symmetry breaking which is given by

$$
\begin{aligned}
\mathrm{SU}\left(N_{v}\right. & \left.+N_{s}\right) \otimes \mathrm{U}(1) \otimes \mathrm{SU}\left(N_{v}+N_{s}\right) \otimes \mathrm{U}(1) \\
& \rightarrow \mathrm{SU}\left(N_{v}+N_{s}\right) \otimes \mathrm{U}(1)
\end{aligned}
$$

for staggered fermions with three colors. Note that in this case the $\mathrm{U}(1)$ symmetry is broken without an anomaly such that the number of Goldstone bosons equals $\left(N_{v}+\right.$ $\left.N_{s}\right)^{2}$. The masses of the flavor non-diagonal mesons $\bar{q}_{i} q_{j}$ $(i \neq j)$ are simply given by $m^{2}=A\left(m_{i}+m_{j}\right) / 2$.

The case of the flavor diagonal mesons is more difficult because we must also consider annihilation according to

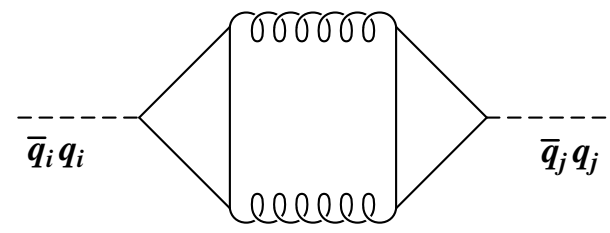

FIG. 2. Annihilation diagram.
Fig 2. Since on the lattice with staggered fermions the broken $U(1)$ symmetry is anomaly free the amplitude of this diagram is proportional to $m_{i} m_{j}$. This is in contrast to the continuum case where the contribution of the diagram remains non-zero even in the chiral limit because of the chiral anomaly. For the mass-squared matrix $M^{2}$ of these states $\left(\bar{v}_{1} v_{1}, \ldots, \bar{v}_{N_{v}} v_{N_{v}}, \bar{s}_{1} s_{1}, \ldots, \bar{s}_{N_{s}} s_{N_{s}}\right)^{T}$ we get in addition to the usual linear terms a contribution that is quadratic in the quark masses,

$$
\begin{aligned}
M^{2}= & A \operatorname{diag}\left(m_{v}, \ldots, m_{v}, m_{s}, \ldots, m_{s}\right) \\
& +z\left(\begin{array}{ccccccc}
m_{v}^{2} & \cdots & m_{v}^{2} & m_{v} m_{s} & \cdots & m_{v} m_{s} \\
\vdots & \ddots & \vdots & \vdots & \ddots & \vdots \\
m_{v}^{2} & \cdots & m_{v}^{2} & m_{v} m_{s} & \cdots & m_{v} m_{s} \\
m_{s} m_{v} & \cdots & m_{s} m_{v} & m_{s}^{2} & \cdots & m_{s}^{2} \\
\vdots & \ddots & \vdots & \vdots & \ddots & \vdots \\
m_{s} m_{v} & \cdots & m_{s} m_{v} & m_{s}^{2} & \cdots & m_{s}^{2}
\end{array}\right)
\end{aligned}
$$

where we have introduced an additional parameter $z$. In contrast to our earlier publication [15] for $\mathrm{SU}(2)$ we cannot neglect this higher-order term here because it will turn out to be the leading order term for $\chi^{\text {conn }}$.

After diagonalization of $M^{2}$ we obtain five different eigenvalues: $A m_{v}, A m_{s}, A\left(m_{v}+m_{s}\right) / 2$ and $\lambda_{ \pm}$with multiplicities given in Table IV. The eigenvalues $\lambda_{ \pm}$are given by

$$
\begin{aligned}
\lambda_{ \pm}=\frac{A}{2}\left(m_{v}+m_{s}\right)+\frac{z}{2}\left(N_{v} m_{v}^{2}+N_{s} m_{s}^{2}\right) \\
\pm \frac{1}{2}\left[A^{2}\left(m_{v}-m_{s}\right)^{2}+z^{2}\left(N_{v} m_{v}^{2}+N_{s} m_{s}^{2}\right)^{2}\right. \\
\left.\quad+2 A z\left(m_{v}-m_{s}\right)\left(N_{v} m_{v}^{2}-N_{s} m_{s}^{2}\right)\right]^{1 / 2}
\end{aligned}
$$

\begin{tabular}{cc}
\hline \hline$m^{2}$ & Multiplicity \\
\hline$A m_{v}$ & $N_{v}^{2}-1$ \\
$A m_{s}$ & $N_{s}^{2}-1$ \\
$A\left(m_{v}+m_{s}\right) / 2$ & $2 N_{v} N_{s}$ \\
$\lambda_{-}$ & 1 \\
$\lambda_{+}$ & 1 \\
\hline \hline
\end{tabular}

TABLE IV. The light particle spectrum for the gauge group $\mathrm{SU}(3)$.

Using Eq. (18) and the multiplicities of Table IV in Eqs. (5) -( 7 ) we obtain

$$
\begin{aligned}
& \sigma\left(m_{v}, m_{s}\right)=C_{0}+C_{c} m_{v}+C_{d} N_{s} m_{s} \\
& -\frac{A N_{s}}{L^{4}} \sum_{p} \frac{1}{2 \hat{p}^{2}+A\left(m_{v}+m_{s}\right)} \\
& -\frac{m_{v} z}{2 L^{4}} \sum_{p} \frac{\left(\hat{p}^{2}+A m_{s}\right)\left(2 \hat{p}^{2}+A m_{v}\right)}{\left(\hat{p}^{2}+A m_{v}\right)^{2}\left(\hat{p}^{2}+A m_{s}+N_{s} z m_{s}^{2}\right)}
\end{aligned}
$$




$$
\begin{gathered}
\chi^{\mathrm{conn}}(m)=C_{c}+\frac{A^{2} N_{s}}{4 L^{4}} \sum_{p} \frac{1}{\left(\hat{p}^{2}+A m\right)^{2}} \\
-\frac{z}{L^{4}} \sum_{p} \frac{\left(\hat{p}^{2}\right)^{2}}{\left(\hat{p}^{2}+A m\right)^{2}\left(\hat{p}^{2}+A m+N_{s} z m^{2}\right)} \\
\chi^{\operatorname{disc}}(m)=C_{d}+\frac{A^{2}}{4 L^{4}} \sum_{p} \frac{1}{\left(\hat{p}^{2}+A m\right)^{2}} \\
+\frac{z^{2} m^{2}}{2 L^{4}} \sum_{p} \frac{\left(2 \hat{p}^{2}+A m\right)^{2}}{\left(\hat{p}^{2}+A m\right)^{2}\left(\hat{p}^{2}+A m+N_{s} z m^{2}\right)^{2}} .
\end{gathered}
$$

In these expressions $C_{0}, C_{c}$, and $C_{d}$ (assumed to be constants) describe the smooth background contributions. For our quenched simulations $\left(N_{s}=0\right)$ the leading $m$ dependent term in the connected scalar susceptibility is proportional to $z$ and thus to the annihilation diagram. This has the consequence that for $\chi^{\text {conn }}$ the $m \rightarrow 0$ limit of chPT does not coincide with the $m \rightarrow \infty$ limit of chRMT anymore. Therefore there is no mass range where both theories coincide.

On the other hand, in the $\mathrm{SU}(2)$ case and for $\chi^{\text {disc }}$ we can identify three mass ranges. Above $m \propto 1 / \sqrt{V}$ chRMT fails since the kinetic terms in the chiral Lagrangian become important. Below $m \propto 1 / V$ chPT becomes invalid because it does not include the nonperturbative contributions to the partition function due to the zero-momentum modes. For sufficiently large volumes there is an overlap region of chRMT and chPT which is shown in Fig. 3.

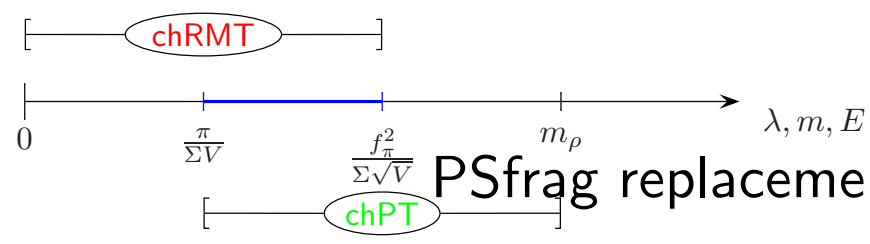

FIG. 3. Energy ranges for the applicability of chiral random matrix theory (chRMT) and chiral perturbation theory (chPT).

In the thermodynamic limit $(L \rightarrow \infty)$ and for $N_{s}=0$ our observables are given by

$$
\begin{aligned}
\sigma(m)= & C_{0}+\left(C_{c}-z a_{0}\right) m-\frac{3 A z}{32 \pi^{2}} m^{2} \ln (A m) \\
& +\mathcal{O}\left(m^{2}\right), \\
\chi^{\operatorname{conn}}(m)= & C_{c}-z a_{0}-\frac{3 A z}{16 \pi^{2}} m \ln (A m)+\mathcal{O}(m), \\
\chi^{\operatorname{disc}}(m)= & -\frac{A^{2}}{64 \pi^{2}} \ln (A m)+C_{d}-\frac{A^{2} a_{1}}{4}-\frac{A^{2}}{64 \pi^{2}} \\
& +\mathcal{O}(m \ln m) .
\end{aligned}
$$

The numerical constants $a_{0}$ and $a_{1}$ take the values $a_{0}=$ 0.1549 and $a_{1}=-0.03035$.
It is interesting to compare these formulae with the corresponding results for quenched SU(2) (see Ref. [15]). In $\mathrm{SU}(2)$ the leading $m$-dependent term in $\sigma$ goes with $m \ln (A m)$, while in quenched $\mathrm{SU}(3)$ this term is absent. Similarly we see that in $\mathrm{SU}(2)$ the leading term in $\chi^{\text {conn }}$ goes with $\ln (A m)$, which is again absent here. So chiral perturbation theory also predicts that in quenched $\mathrm{SU}(3)$ the generic leading term, found in $\mathrm{SU}(2)$ and dynamical $\mathrm{SU}(3)$, is missing. This is the same sort of result that we saw in chRMT in Eq. (17).

What is the physical reason for this difference? Chiral perturbation theory lets us understand the cause. In Fig. 1 we show the simplest Goldstone meson contributions to $\chi^{\text {conn }}$ and $\chi^{\text {disc }}$. The $\times$ represents a $\bar{\psi} \psi$ operator. In $\chi^{\text {conn }}$ both operators are on the same quark line, in $\chi^{\text {disc }}$ they are always on different quark lines (this is the reason for the nomenclature). We see that the diagram for $\chi^{\text {disc }}$ has no spectator quark loops, so it survives when we take the quenched limit. The diagram for $\chi^{\text {conn }}$ has a spectator loop, and so it vanishes in the quenched approximation, when all spectator loops are ignored. Why then does $\chi^{\text {conn }}$ for quenched SU(2) still have a logarithmic term? This is because, as emphasized in [15], $\mathrm{SU}(2)$ has "Goldstone baryons" as well as Goldstone mesons. These are two-quark states with masses that vanish in the chiral limit, just like the more familiar Goldstone bosons of the other $\mathrm{SU}\left(N_{c}\right)$ groups. In Fig. 5 we sketch a Goldstone baryon contribution to $\chi^{\text {conn }}$ which survives in the quenched approximation.

$$
\chi^{\mathrm{conn}} \quad \chi^{\mathrm{disc}}
$$
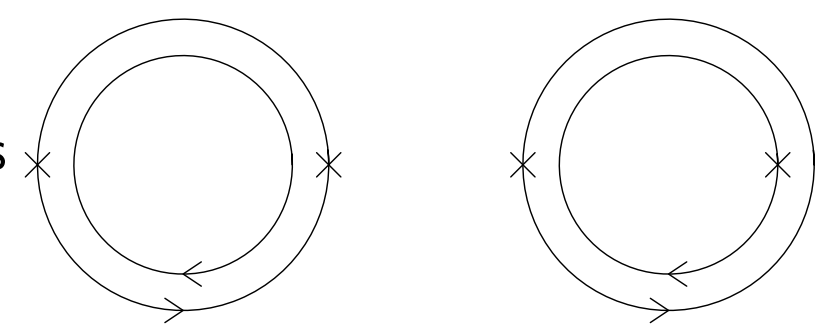

FIG. 4. Goldstone meson contribution to $\chi^{\text {conn }}$ and $\chi^{\text {disc }}$.

$$
\chi^{\mathrm{conn}}
$$

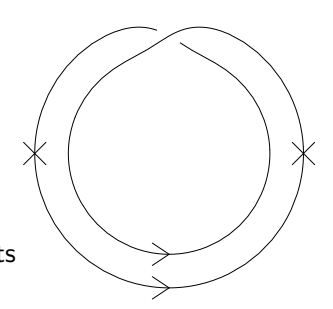

FIG. 5. Goldstone baryon contribution to $\chi^{\text {conn }}$ for gauge group $\mathrm{SU}(2)$. 


\begin{tabular}{ccccc}
\hline \hline$\beta$ & $A$ & $B$ & $C_{d}$ & $f_{\pi}$ \\
\hline 5.2 & $5.9(9)$ & $1.5(16)$ & $-1.6(2)$ & $0.30(3)$ \\
5.4 & $7.2(9)$ & $0.55(4)$ & $-3.4(12)$ & $0.25(2)$ \\
5.6 & $10.0(18)$ & $0.29(5)$ & $-7.0(33)$ & $0.15(2)$ \\
5.7 & $7.8(15)$ & $0.11(4)$ & $-4.2(21)$ & $0.13(3)$ \\
\hline \hline
\end{tabular}

TABLE V. The fit parameters $A, B$, and $C_{d}$ as well as the values of $f_{\pi}$ obtained from Eq. (29).

\section{COMPARISON WITH LATTICE DATA}

We want to confront the lattice data with the predictions from chPT just described concentrating solely on the disconnected chiral susceptibility $\chi^{\text {disc }}(m)$ because for the other two quantities there is no common range of applicability of chRMT and chPT. Since the terms proportional to $z$ are of higher order we will neglect them in the following. It is important to note that besides the Goldstone boson $\pi$ the meson spectrum contains also 15 would-be Goldstone bosons whose masses do not vanish in the chiral limit $m \rightarrow 0$. (Remember that one generation of staggered fermions corresponds to four flavors in the continuum limit.) Instead we expect their masses $m_{i}$ to depend on $m$ according to $m_{i}^{2}=A_{i} m+B_{i}$. They all contribute a term of the form $\frac{A_{i}^{2}}{4 L^{4}} \sum_{p}\left(\hat{p}^{2}+A_{i} m+B_{i}\right)^{-2}$. Besides the Goldstone boson $\pi$ (with $B_{\pi}=0$ ) the theory would allow for up to 7 different boson masses [19]. Since we cannot afford so many fit parameters we introduce just a single "effective" $B$ for the 15 would-be Goldstone bosons and set $A_{i}=A$. The susceptibility $\chi^{\text {disc }}$ then becomes

$$
\begin{aligned}
\chi^{\mathrm{disc}}(m)=C_{d}+\frac{A^{2}}{4 L^{4}} \sum_{p} & {\left[\frac{1}{\left(\hat{p}^{2}+A m\right)^{2}}\right.} \\
& \left.+15 \frac{1}{\left(\hat{p}^{2}+A m+B\right)^{2}}\right] .
\end{aligned}
$$

The parameters $A, B$, and $C_{d}$ have been fitted jointly for all lattice sizes that are available for our particular values of $\beta$. The parameters are, in principle, functions only of $\beta$ but not of $L$. The fit interval has been chosen such that its left border is within the overlap region of chRMT and chPT where the data show the asymptotic behavior of both theories. The large- $m$ cutoff is more difficult, because one has to be careful not to extend the fit into regions where our version of chPT is not applicable anymore. We therefore extended the interval until we found a stable plateau of the parameter $A$.

The results for the fit parameters are given in Table $\mathrm{V}$. One observes a monotonic decrease of the parameter $B$ as $\beta$ gets larger. This shows that the would-be Goldstone bosons become more and more important when one approaches the continuum limit. Such a behavior is of course expected because in the continuum limit all 16 (would-be) Goldstone bosons should have the same
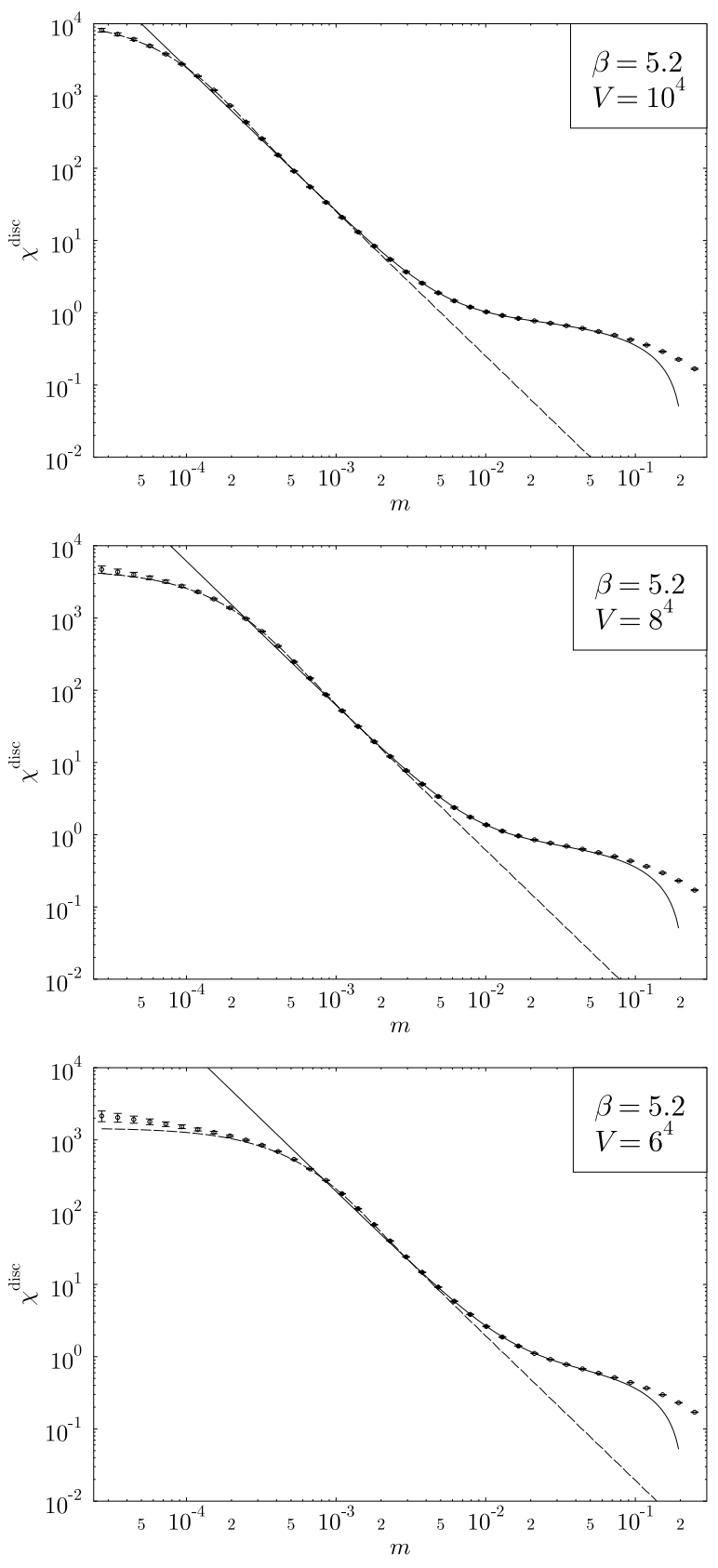

FIG. 6. The chiral susceptibility $\chi^{\text {disc }}$ at $\beta=5.2$ for $L=6,8,10$ plotted against $m$. The dashed curve represents the chRMT prediction. The full curve results from a joint fit of all lattice volumes with the chPT formula (28). The fit parameters are given in Table $\mathrm{V}$.

mass, at least as far as quenching artifacts can be neglected. The parameter $A$, on the other hand, shows some indication of non-monotonicity. This is also not completely unexpected: Strong coupling calculations 20] show that $m_{\pi}^{2} / m$ has a value of about 4.6 at $\beta=0$ and increases as $\beta$ grows. But for $\beta \rightarrow \infty$ the ratio $m_{\pi}^{2} / m$ having dimension of a mass has to decrease to zero in ac- 

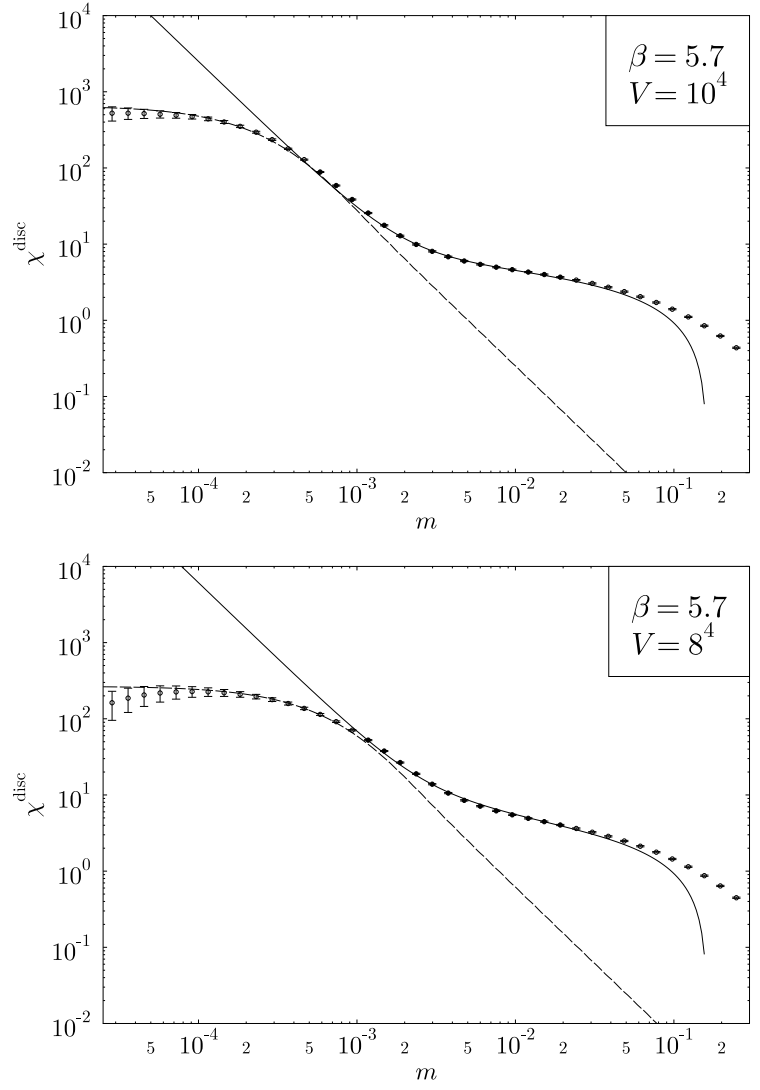

FIG. 7. The chiral susceptibility $\chi^{\text {disc }}$ at $\beta=5.7$ for $L=8,10$ plotted against $m$. The meaning of the curves is the same as in Fig. 6. The fit parameters are given in Table $\mathrm{V}$.

cordance with the renormalization group. Although the strong coupling expansions involve dynamical fermions, one would not be surprised to find a similar behavior in quenched simulations.

In Figs. 6 and 7 we show our data for $\chi^{\text {disc }}$ together with the chRMT prediction and the chPT fits at $\beta=$ 5.2 and 5.7, respectively. This double logarithmic plot against $m$ confirms the expectation (see Fig. 3) that the range (in $m$ ) of common applicability of chRMT and chPT increases with $L$. Indeed we read off from Fig. 3 that the ratio of the upper end of this range over the lower end is roughly given by $f_{\pi}^{2} L^{2} / \pi$. In Fig. 8 we compare the $L=10$ data for our different $\beta$ values. We plot $\chi^{\text {disc }} /\left(V \Sigma^{2}\right)$ against $u=m V \Sigma$ together with the chRMT prediction and the chPT fits. The range of common applicability of chRMT and chPT decreases as $\beta$ grows, because $f_{\pi}$ (in lattice units) must tend to zero in the continuum limit.

The link with usual chPT is provided by the GellMann-Oakes-Renner relation that relates the parameter $A$ to $\Sigma$ and the pion decay constant $f_{\pi}$,

$$
A=\frac{2}{f_{\pi}^{2}} \frac{\Sigma}{4} .
$$
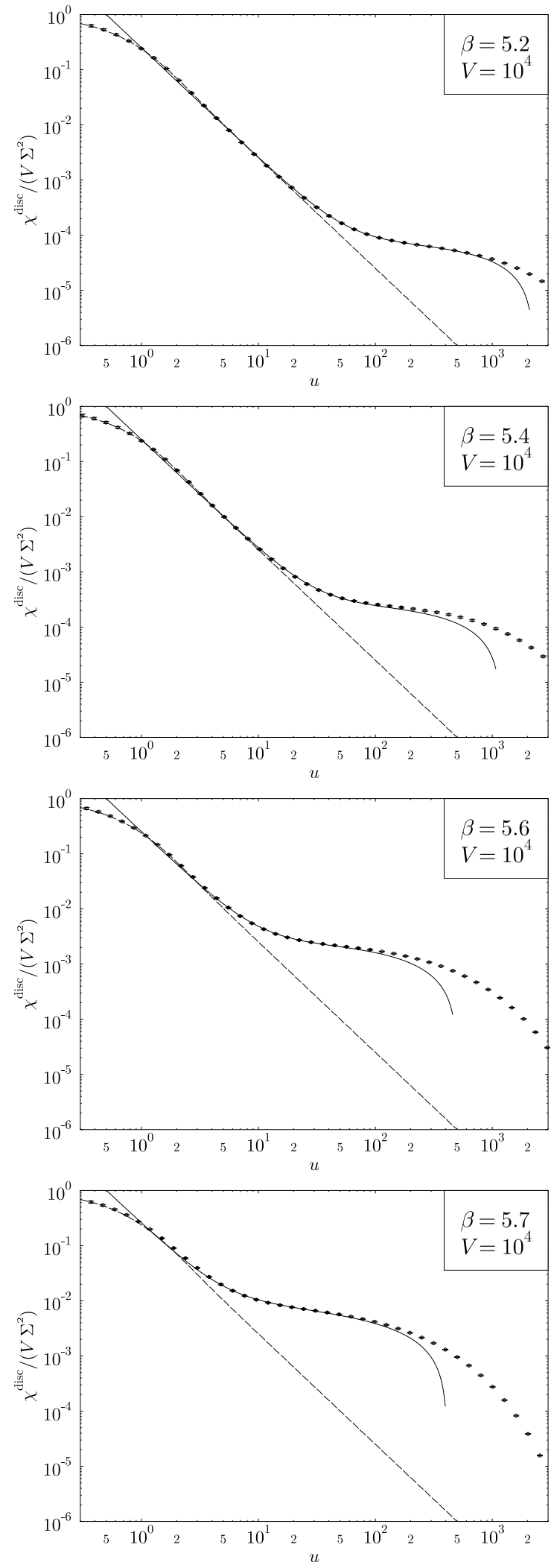

FIG. 8. The chiral susceptibility $\chi^{\text {disc }} /\left(V \Sigma^{2}\right)$ for $L=10$ and $\beta=5.2,5.4,5.6,5.7$ plotted against $u=m V \Sigma$. The meaning of the curves is the same as in Fig. 6. The fit parameters are given in Table $\mathrm{V}$. 
The (unrenormalized) values of $f_{\pi}$ calculated from this equation are shown in Table $\mathrm{V}$.

In Ref. 21 Goldstone boson masses $m_{\pi}$ have been computed with quenched staggered fermions on a $16^{3} \times 32$ lattice at $\beta=5.7$. Fitting these masses with the relation $m_{\pi}^{2}=A_{\pi} m+B_{\pi}$ the authors find $A_{\pi}=7.96(5)$ and $B_{\pi}=0.004(1)$. Our value for $A$ at $\beta=5.7$ agrees very well with this result giving us confidence that our chPT model captures the essential features of the underlying physics.

\section{SUMMARY}

In this paper we have studied spectral properties of the staggered Dirac operator in quenched SU(3) lattice gauge theory in the phase where chiral symmetry is spontaneously broken. From complete spectra of the Dirac operator we have computed the scalar susceptibilities $\chi^{\text {disc }}$ and $\chi^{\text {conn }}$ as functions of the (valence) quark mass $m$. For small masses the low-lying eigenvalues of the Dirac operator give the dominant contribution and the mass dependence follows the predictions of chiral random matrix theory. This agreement holds for masses which are smaller than the so-called Thouless energy. In the generic case, the Thouless energy scales like $L^{2}$, where $L$ is the linear size of the lattice. In physical terms, this behavior results from the fact that the Compton wavelength of the lightest particles in the theory, the Goldstone bosons, exceeds $L$ as long as $m$ lies below the Thouless energy, and the susceptibilities are therefore insensitive to the details of the dynamics. For the gauge group SU(2) the expected scaling behavior has been confirmed previously for both susceptibilities 14,15. In the present case of gauge group $\mathrm{SU}(3)$ we observed scaling with $L^{2}$ for $\chi^{\text {disc }}$ [10], whereas for $\chi^{\text {conn }}$ the Thouless energy was found to scale with $L^{4 / 3}$. This exceptional behavior is explained as a quenching artifact.

Above the Thouless energy the Goldstone bosons begin to fit into the lattice volume and one enters the realm of (quenched) chiral perturbation theory. The application of chiral perturbation theory to our case is complicated by the subtle chiral properties of staggered fermions. In particular, it turns out that for our simulation parameters the contributions from the would-be Goldstone bosons cannot be neglected. Taking them into account by means of a rough model we obtain a satisfactory description of our data, which also allows us to determine the pion decay constant $f_{\pi}$. At our largest $\beta$ value $(\beta=5.7)$ we could compare our result for $f_{\pi}$ with numbers from the literature and found nice agreement.

After completion of our work, a preprint 22] appeared that discusses related issues using (partially) quenched chiral perturbation theory, also in a finite volume but already in the continuum limit.

\section{ACKNOWLEDGMENTS}

This work was supported by DFG, BMBF, DOE contracts DE-FG02-91ER40608 and DE-AC02-98CH10886, and the RIKEN-BNL Research Center.

[1] E.V. Shuryak and J.J.M. Verbaarschot, Nucl. Phys. A560, 306 (1993).

[2] For a recent review on random matrix theory in general, see T. Guhr, A. Müller-Groeling, and H.A. Weidenmüller, Phys. Rep. 299, 189 (1998) and references therein.

[3] For recent reviews on chRMT and QCD Dirac spectra, see J.J.M. Verbaarschot and T. Wettig, Annu. Rev. Nucl. Part. Sci. 50, 343 (2000); J.J.M. Verbaarschot, hep$\mathrm{ph} / 9902394$ and references therein.

[4] M.A. Halasz and J.J.M. Verbaarschot, Phys. Rev. Lett. 74, 3920 (1995).

[5] R.G. Edwards, U.M. Heller, and R. Narayanan, Phys. Rev. D 60, 077502 (1999).

[6] M.E. Berbenni-Bitsch, S. Meyer, A. Schäfer, J.J.M. Verbaarschot, and T. Wettig, Phys. Rev. Lett. 80, 1146 (1998).

[7] J.-Z. Ma, T. Guhr, and T. Wettig, Eur. Phys. J. A 2, 87 (1998).

[8] M.E. Berbenni-Bitsch, S. Meyer, and T. Wettig, Phys. Rev. D 58, 071502 (1998).

[9] P.H. Damgaard, U.M. Heller, and A. Krasnitz, Phys. Lett. B 445, 366 (1999).

[10] M. Göckeler, H. Hehl, P.E.L. Rakow, A. Schäfer, and T. Wettig, Phys. Rev. D 59, 094503 (1999).

[11] J.J.M. Verbaarschot, Phys. Lett. B 368, 137 (1996).

[12] J.C. Osborn and J.J.M. Verbaarschot, Phys. Rev. Lett. 81, 268 (1998); Nucl. Phys. B525, 738 (1998).

[13] R.A. Janik, M.A. Nowak, G. Papp, and I. Zahed, Phys. Rev. Lett. 81, 264 (1998).

[14] M.E. Berbenni-Bitsch, M. Göckeler, T. Guhr, A.D. Jackson, J.-Z. Ma, S. Meyer, A. Schäfer, H.A. Weidenmüller, T. Wettig, and T. Wilke, Phys. Lett. B 438, 14 (1998).

[15] M.E. Berbenni-Bitsch, M. Göckeler, H. Hehl, S. Meyer, P.E.L. Rakow, A. Schäfer, and T. Wettig, Phys. Lett. B 466, 293 (1999).

[16] P.J. Forrester, Nucl. Phys. B402, 709 (1993).

[17] M.E. Berbenni-Bitsch, M. Göckeler, H. Hehl, S. Meyer, P.E.L. Rakow, A. Schäfer, and T. Wettig, Nucl. Phys. Proc. Suppl. 83-84, 974 (2000).

[18] P.H. Damgaard, U.M. Heller, R. Niclasen, and K. Rummukainen, Phys. Rev. D 61, 014501 (2000).

[19] M.F.L. Golterman, Nucl. Phys. B273, 663 (1986).

[20] T. Jolicœur, H. Kluberg-Stern, M. Lev, A. Morel, and B. Petersson, Nucl. Phys. B235, 455 (1984).

[21] R. Gupta, G. Guralnik, G.W. Kilcup, and S.R. Sharpe, Phys. Rev. D 43, 2003 (1991).

[22] P.H. Damgaard, hep-lat/0105010. 